Arts

et Savoirs

\section{Arts et Savoirs}

13 | 2020

Lumières plurielles

\title{
Direction et éducation genrée dans Les Lettres du pape Clément XIV de Caraccioli
}

Spiritual direction and gender education in Interesting Letters of the Late Pope

Clement XIV (Ganganelli) by Caraccioli

\section{Stéphanie Géhanne Gavoty}

\section{(2) OpenEdition}

\section{Journals}

Édition électronique

URL : http://journals.openedition.org/aes/2611

DOI : 10.4000/aes.2611

ISSN : 2258-093X

Éditeur

Laboratoire LISAA

Référence électronique

Stéphanie Géhanne Gavoty, « Direction et éducation genrée dans Les Lettres du pape Clément XIV de

Caraccioli », Arts et Savoirs [En ligne], 13| 2020, mis en ligne le 01 juin 2020, consulté le 19 juin 2020. URL : http://journals.openedition.org/aes/2611 ; DOI : https://doi.org/10.4000/aes.2611

Ce document a été généré automatiquement le 19 juin 2020.

Centre de recherche LISAA (Littératures SAvoirs et Arts) 


\title{
Direction et éducation genrée dans Les Lettres du pape Clément XIV de Caraccioli
}

\author{
Spiritual direction and gender education in Interesting Letters of the Late Pope \\ Clement XIV (Ganganelli) by Caraccioli
}

Stéphanie Géhanne Gavoty

1 C'est d'un bon mot de Grimm qui proposait de marier Louis-Antoine de Caraccioli avec $\mathrm{M}^{\mathrm{me}}$ Leprince de Beaumont ${ }^{1}$ qu'est venue l'idée d'examiner, sous l'angle de la pédagogie, l'œuvre la plus fameuse de Caraccioli, les Lettres intéressantes du pape Clément $X I V^{2}$, magistère épistolaire publié avec grand succès en décembre 1775 , un peu plus d'un an après le décès du pape (septembre 1774), dans des circonstances fumeuses et dans un contexte religieux grave, celui de la suppression de la Compagnie de Jésus en 1773.

2 Deux articles au moins ont déjà été consacrés au pédagogue Caraccioli, celui de Martine Jacques, au cours du colloque organisé par Nicolas Brucker en 2008, et celui de Maximilian Gröne, à Augsburg en $2012^{3}$. Ils montrent que ce littérateur, qui fut régent de basse classe au collège de l'Oratoire à Vendôme, précepteur des fils d'un magnat polonais, l'auteur d'un Véritable Mentor en 1756, plusieurs fois réédité et traduit, et celui de La Vraie manière d'éduquer les princes publié à l'aube de la Révolution, fut toute sa vie un éducateur ${ }^{4}$.

D'autres études ${ }^{5}$ ont établi que Caraccioli s'inscrit pleinement dans le courant de démocratisation des savoirs, lui qui sut toucher un large public, notamment les femmes de la bourgeoisie aisée, ayant récemment accédé à la lecture, ou la clientèle plus modeste des loueurs de livres.

4 La vulgarisation se traduit en outre sur le plan des savoirs, puisque Caraccioli a manifesté avec constance une volonté de simplification. Celle-ci le conduit à évacuer un bagage théologique qu'il a vraisemblablement possédé, le réduisant sciemment pour porter d'abord son effort sur les pré-requis ou éléments, dans le but de mettre à la 
portée de tous ceux qui constituent alors le lectorat émergent une instruction chrétienne qui leur soit accessible.

5 Cette préoccupation pédagogique se retrouve dans les formes auxquelles Caraccioli a pu recourir, bien conscient qu'un message de portée similaire ne sera pas transmis de la même façon selon les publics. L'ensemble de sa production est marqué par cette souplesse des formes adaptées au lectorat ciblé : entretiens dispensés au soir tombé dans les Derniers adieux de la maréchale à ses enfants, dictionnaires, recueils épistolaires, voire romans.

6 C'est l'un de ces recueils épistolaires qui nous occupe ici, cette étonnante hagiographie, à la gloire de Clément XIV, peint sous les traits du Ganganelli philosophe qu'il ne fut sans doute pas, au travers de lettres en bonne part fictives que donna Caraccioli, orchestrant un véritable coup éditorial, décliné en biographie, supplément aux Lettres, mais aussi réponses aux détracteurs quand ceux-ci se firent plus audibles ${ }^{6}$.

Qui lit les Lettres intéressantes du pape Clément XIV est en effet frappé par l'écriture sapientiale qui s'y déploie lettre après lettre, selon une partition agréablement orchestrée. Ganganelli y prodigue, avec force impératifs, conseils, exhortations, objurgations, et se positionne en directeur pour de nombreux correspondants. Thèmes et style se conjuguent pour forger en maître à penser celui qui sera appelé à régner : un maître à penser, tant dans l'ordre religieux que dans les affaires domestiques, un savant qui n'est pas un érudit stérile, mais un homme sage et pragmatique. Nous verrons sous peu le détail, d'autant plus intéressant que parmi les cent trente-deux lettres du recueil initial (auquel nous limiterons notre propos ${ }^{7}$ ), certaines abordent notablement des questions d'éducation, invitant le chercheur à interroger le positionnement (éventuellement genré) des prescriptions qui s'y trouvent, point que la seconde partie envisagera.

8 Mais au-delà des thématiques, présentées et commentées dans la première partie, il faut considérer aussi l'effet-personnage ${ }^{8}$, particulièrement significatif lorsque la matière est une de ces mystifications si brillamment analysées par J.-F. Jeandillou ${ }^{9}$ : une tradition facile à établir atteste, de longue date, la connexion entre le saint homme, l'épistolier et l'éducateur. Saint Jérôme ${ }^{10}$, Saint François de Sales ${ }^{11}$, Fénelon écrivirent des lettres riches de leurs conseils avisés. Bref, la posture est de haute tradition. C'est pourquoi nous nous attacherons à montrer que la figure du maître est une composante essentielle de la mystification, de sa réussite comme de son éventement.

Le recueil clémentin donnait à lire des lettres philosophiques et familières, selon la distinction de Formey : celles « qu'on peut appeler philosophiques, où l'on traite d'une manière libre quelques sujets appartenant aux sciences ou à la littérature; les autres familières, qui ne sont autre chose qu'une espèce de conversation entre des absents. " $^{12}$

La correspondance du moine Ganganelli ou celle du cardinal qu'il est devenu combinent, d'une lettre à l'autre ou au sein d'une même missive, ces deux dimensions. Cela est vrai des lettres adressées à des religieux de tous ordres, parfois membres de la Curie, de celles destinées à des personnalités de la République des lettres ou des sciences, de celles enfin adressées à des particuliers tels un jeune comte, fils d'un ami défunt (que le bon moine tâche de ramener dans le droit chemin par une direction 
adaptée au profil et fort zélée), ou une mère de famille anonyme qui l'a interpelé sur l'éducation de ses filles.

11 Ces destinataires, qui relèvent du clergé ou du monde, fonctionnent en miroir avec le lectorat effectif de Caraccioli. Nous avons établi, dans notre thèse de doctorat, une typologie des lecteurs des Lettres du pape Clément XIV, lectorat assurément mixte (comme il se doit de tout best-seller) composé de religieux, de notables de province, hommes et femmes, de portiers d'hôtel, de jeunes filles telle la future $\mathrm{M}^{\text {me }}$ Roland, de jeunes gens qui, se faisant « l'écho du sexe », ont trouvé les Lettres « admirables »"

Ce public hétérogène était explicitement visé dans l'Avertissement qui précède la première édition des Lettres :

L'homme du cloitre, l'homme du monde, l'homme de cour, les mères de famille, les religieuses, les jeunes gens, les vieillards, y puiseront des principes lumineux, des conseils utiles; et il n'y a point de père éclairé, lorsqu'il les aura lues, qui ne mette en pratique les préceptes qu'on y trouve sur l'éducation. ${ }^{14}$

"Intéressantes ", ces Lettres l'étaient assurément par la variété des sujets abordés, que les journalistes ne manquèrent pas de souligner pour la louer ou s'en moquer. Tel ce détracteur de l'ouvrage, le père Bonnaud qui les raillait en désignant Caraccioli pour l'auteur véritable des

Lettres politico - mathématico - physico - géographico - botanico - médico théologico - historico - critico - pittorico - poético - bibliographico - philosophico - métaphysico - économico - philologico - épigrammatico - et par-dessus le marché, prophético - intéressantes, attribuées au pape Clément XIV. ${ }^{15}$

La diversité des correspondants de Ganganelli autorisait cette variété des objets. Les religieux avec lesquels il est en liaison sont l'occasion d'aborder des thématiques qui relèvent d'un magistère chrétien, destiné à enseigner les catholiques de profession, à les confirmer dans leur foi, à préciser leur doctrine ou leurs devoirs, en somme à forger une identité commune autour de valeurs et de principes partagés. Il nous semble que ces "conseils utiles» relèvent de la direction, au sens grégorien du terme regimen animarum, le " gouvernement des âmes ", entendu selon des applications pratiques qui varient au gré des correspondants ${ }^{16}$. Bien évidemment, direction et éducation n'ont pas les mêmes visées : le directeur doit inspirer au chrétien l'humilité, et non pas cultiver son esprit, ce qui est le rôle conjoint du précepteur et du gouverneur ${ }^{17}$.

Ganganelli prodigue ainsi une série de sept avis à un directeur de religieuses, dont le suivant, qui nous intéressera dans une perspective genrée : «Ayez égard à la faiblesse d'un sexe qui exige de la condescendance dans la manière de le gouverner. Il faut de l'indulgence pour de pauvres recluses, chez qui l'imagination travaille $»^{18}$. Dans la quatre-vingt-neuvième lettre, Ganganelli livre, à la demande de son correspondant, des avis propres à en faire un confesseur exemplaire ${ }^{19}$. Direction et éducation se mêlent dans la lettre CIII : Ganganelli y déploie, à l'attention d'un religieux en partance pour l'Amérique, un véritable plan d'études, vade mecum pour cultiver son esprit avec méthode et humilitée 2 .

L'intérêt de telles lettres est qu'elles mettent en place, s'agissant de la figure de Ganganelli, un ethos de conseiller, placé de facto dans une position de supériorité (doctorale ou spirituelle) par rapport au conseillée ${ }^{21}$. Le binôme conseiller-conseillé est un avatar de la relation maître-élève, « instructeur-instructaire ", néologisme décalqué de destinateur-destinataire. 
17 En outre, la direction de moines peut être envisagée comme l'équivalent, dans l'ordre ecclésial, de l'éducation dans l'espace séculier. De fait, les Lettres du pape Clément XIV offrent une variation laïque sur la thématique de la direction, en donnant à lire trois lettres qui traitent exclusivement de l'éducation, pièces susceptibles d'intéresser le lectorat profane de Caraccioli dont la passion pédagogique n'est plus à démontrer ${ }^{22}$.

Il s'agit d'abord de l'éducation des filles dans la lettre LV, comme suite à la requête de la dame Picliani, désireuse de connaître les vues du sage Ganganelli, puis de celle des garçons dans une longue lettre adressée à un gentilhomme de Toscane (lettre LXXIV) ${ }^{23}$. La lettre CIII, déjà mentionnée, est quant à elle un traité de l'organisation des études qui forme une quinzaine de pages.

Pour Caraccioli, « l'éducation est l'art des arts ${ }^{24}$. Ces lettres pédagogiques comptaient donc pour le pseudo-éditeur qui les signalait dès la préface. Lorsqu'il compile les quatre volumes de Lettres pour en donner un abrégé, les Pensées du pape Clément XIV, ces lettres se trouvent presque intégralement reproduites (avec quelques coupes et aménagements stylistiques) aux entrées : «Éducation », "Esprit d'invention », "État. Choix d'un état », "Étude ». Or, « l'extrait a une efficacité pédagogique certaine : il rend plus accessible les œuvres imposantes $»^{25}$. L'extrait sélectionne la quintessence et ainsi l'indique à l'œil non aguerri.

Ces trois missives, que la compilation met en avant, sont redoublées, au sein du recueil, par une série de lettres (une vingtaine) dans lesquelles Ganganelli passe de la théorie à la pratique $d u$ "gouvernement des jeunes âmes ", en ramenant dans le droit chemin ce jeune comte qui s'en était écarté. Ainsi la lettre XXX, la troisième de la série, offre-t-elle à lire un plan de conversion ${ }^{26}$ pour cet enfant auquel Ganganelli a donné les "premières instructions ${ }^{27}$. De manière classique, dans les romans épistolaires ou dans les recueils de lettres supposées, la lettre est écrite à la demande du destinataire («Puisque vous voulez un plan de ma main pour vous guider [...] $»^{28}$ ). Cette requête autorisera le pseudo-Ganganelli à délivrer ses préceptes, dressant d'abord un plan de conduite (lettre XXX), puis une bibliothèque (lettre XL), comme d'autres avant ou après lui (les abbés Pluche, Gérard, Reyre, $\mathrm{M}^{\mathrm{me}}$ de Genlis, etc.) ${ }^{29}$.

21 L'intérêt de cette série, véritable petit « roman de direction » d'après le rédacteur du Tartuffe épistolaire démasqué $e^{30}$, est qu'elle permet de joindre les préceptes à la pratique, mais aussi le général au particulier. C'est avec douceur et fermeté que Ganganelli guide le jeune homme. Ainsi, les conseils qu'il prodigue à ses autres correspondants lorsqu'il s'agit d'éducation sont parfaitement actualisés (et donc répétés) dans cette série épistolaire. Leur efficacité est également prouvée par un heureux dénouement.

Un dernier point doit être encore souligné. Dans l'abrégé, les entrées relatives à l'éducation côtoient celles intitulées "Religion ", "Dévotion véritable ", " Papes ", cette promiscuité confirmant chez Caraccioli la contiguïté de l'éducation et de la religion, comme le soulignait déjà Maximilian Gröne: ainsi c'est au "gouverneur éclairé » qui présidera à l'éducation du prince ou à celle des jeunes Toscans, et non au prêtre, «de prendre soin de la formation morale, c'est-à-dire chrétienne, de son disciple. ${ }^{31}$ Nous y reviendrons dans la seconde partie.

Ainsi la collection souscrit-elle pleinement à la passion pédagogique des lecteurs. La diversité des correspondants de ce recueil monodique permet d'offrir un kaléidoscope de situations, qui sont autant d'avatars d'une relation maître-disciple, conseillerconseillé, déclinée ici dans la sphère religieuse, plus spécifiquement romaine qu'appelle 
un tel destinateur, et donnant lieu à des épîtres chrétiennes professant, sous couvert de conseils, des principes de gouvernement. La relation s'exprime aussi dans la sphère laïque en abordant le champ de l'éducation des enfants, du choix d'un précepteur à celui d'un état pour prodiguer les principes d'une éducation éclairée par la foi catholique et toujours rivée à elle. Elle prend une tonalité plus personnelle lorsqu'il s'agit d'assurer la conversion d'un jeune noble égaré, dont le cheminement acte, par son heureuse résolution, les talents du sage gouverneur qu'est Ganganelli. L'ensemble forme une constellation que centralise la figure de Ganganelli, devenu le pape clément XIV, bien campé dans son rôle de maître à penser.

Que dit ce maître à penser lorsque son magistère se porte sur l'éducation? Qu'en est-il du lieu des études? de la nature de celles-ci ? des rôles respectifs assignés à chaque sexe ? Est-il possible d'y repérer les signes d'une éducation éclairée, ou du moins libérale, correspondant à la figure d'un pape ouvert aux Lumières que Caraccioli entendait incarner en Ganganelli ${ }^{32}$ ? N'y trouve-t-on pas plutôt un compendium pédagogique, d'une part parce qu'il est avéré que «Caraccioli butine sans vergogne et fait son miel de bien des pensées ${ }^{33}$, d'autre part parce que la cible plurielle de son entreprise infère une voie médiane susceptible de plaire au plus grand nombre?

Des éléments de réponse seront apportés par la lecture des deux lettres sur l'éducation que le pseudo-Ganganelli adresse à une dame italienne, la lettre LV, reproduite à la fin de cet article, et celle qu'il adresse à un gentilhomme de Toscane, la soixantequatorzième.

Sans originalité, Caraccioli, en donnant deux lettres en diptyque, distingue les sexes, sans que cette partition ne soit justifiée puisqu'elle va alors de soi $^{34}$.

Qu'en est-il d'abord du lieu des études ? Le premier paragraphe de la lettre LV évoque à demi-mot le modèle conventuel, auquel la dame italienne n'a manifestement pas souscrit. Il serait hâtif d'y percevoir le signe d'une éducation éclairée, les historiens s'accordant pour dater de la fin du xvir è siècle l'émergence d'un fort courant de critiques contre l'éducation conventuelle des jeunes filles ${ }^{35}$. La littérature romanesque se fera par ailleurs le relais de cette idée ${ }^{36}$.

S'agissant des études masculines, le pseudo-Ganganelli reproduit, dans la lettre LXXIV, le modèle à l'œuvre dans Le Véritable Mentor, celui de l'éducation "privée " (et non publique) de la noblesse. En effet, il ne faut pas se laisser leurrer par une phrase toute en balancements comme celle-ci : "Il y a bien des raisons qui m'engagent à vous conseiller l'éducation domestique, et il y en a encore plus qui m'empêchent de vous le persuader. $\|{ }^{37}$ L'éducation publique serait moins monotone et favoriserait l'émulation. Pourtant ce sont des préceptes relatifs à l'éducation privée que Ganganelli délivre par la suite, en développant l'idée que son correspondant trouve « un précepteur [...] doux, patient, sociable, éclairé ${ }^{38}$.

29 La question de la nature des études n'offre pas plus de prise à une pédagogie éclairée. En effet, les premiers mots de la lettre LV reconduisent une image bien établie, celle de la mère chrétienne élevant ses enfants opposée à la femme savante, toujours dénigrée ${ }^{39}$. La fin du septième paragraphe est à cet égard éloquente : «lorsqu'il sera question de s'appliquer, vous ne parlerez ni de ces études profondes, ni de ces sciences abstraites, qui souvent rendent le sexe vain et jaseur $»^{40}$. «À petite fille, petites science », écrivait Martine Sonnet, en tête d'un chapitre de son livre, L'éducation des filles au temps des Lumières $^{41}$. Remarquons qu'il n'y a encore rien d'original dans cette négligence de 
l'éducation intellectuelle féminine bien attestée dans les modèles qui président alors à l'éducation des demoiselles ${ }^{42}$.

Le pseudo-Ganganelli n'ôte pas pour autant son admiration aux femmes savantes de l'académie de Bologne (comme il le fait dans la deuxième lettre du recueil ${ }^{43}$ ) ou à la princesse Catherine Barbarigo, cette illustre Vénitienne qui traduisit Locke en italien ${ }^{44}$. Ces effets de contrepoint confirment le sentiment d'une voie médiane, offrant à lire une ligne directrice traditionnelle et quelques concessions à la modernité.

31 A contrario, dans la lettre sur l'éducation des garçons, le pseudo-Ganganelli invite le père à exciter dans ses fils « le plus vif désir de savoir, et la plus forte appréhension de rester ignorants $»^{45}$. Il s'attarde ensuite sur trois domaines, l'histoire, la poésie et l'éloquence. Le programme de Ganganelli rappelle moins les préconisations d'un Locke ou d'un Rousseau quant au travail manuel ${ }^{46}$ que celles de Bérulle ou du père Lamy, membres éminents de l'Oratoire, dont la pédagogie était novatrice ${ }^{47}$, ou encore celles de Fénelon, dont les écrits ont fortement inspiré Caraccioli ${ }^{48}$. Ce programme évoque encore les conseils d'un Rollin qui se soucie essentiellement de l'acquisition du jugement historique et qui, comme Caraccioli, oriente toujours l'étude de l'histoire " selon la causalité transcendante du Dieu de l'alliance » : les causes humaines, causes secondes, révèlent l'action puissante de la Providence ${ }^{49}$. Soulignons enfin qu'il n'est pas question de faire des jeunes gens des docteurs capables de soutenir une thèse, mais de développer l'acquisition des connaissances, la justesse du jugement, la rectitude de la conduite, trois finalités préconisées par le père Lamy dans son programme d'enseignement, mais que partagent bien des pédagogues.

32 Toutefois, plus que les savoirs (l'instruction) importe l'éducation morale, c'est-à-dire la formation à la vie chrétienne. Sur ce point, les recommandations de Ganganelli ne sont pas "genrées »! Dans la lettre LV, il invite d'emblée à ne pas récuser le monde, selon une tradition qui va de saint François de Sales à Fénelon et que l'on retrouve chez $\mathrm{M}^{\mathrm{me}}$ de Maintenon pour laquelle une « religion bien tempérée doit s'accompagner de vertus mondaines $»^{50}$. La lettre LXXIV, qui s'ouvre en rappelant que l'éducation n'est que « vernis, si elle n'est appuyée sur la religion $~^{51}$ parce que Dieu est « le principe et la fin de toutes choses $»^{52}$, se poursuit en mettant en garde contre les pratiques minutieuses ${ }^{53}$. Le motif est celui de la bonne dévotion assorti de la critique de la fausse, leitmotiv de ces lettres $^{54}$ - comme de l'œuvre de Caraccioli - et lieu commun du discours moral à l'âge classique ${ }^{55}$. Le thème est l'expression de la recherche d'une " voie moyenne de foi raisonnable », quête qui est celle des «Lumières religieuses" si l'on s'en tient aux analyses des historiens anglo-saxons ${ }^{56}$.

33 Cette insistance sur la formation de la jeune personne à la civilité chrétienne se justifie par l'autre préoccupation constante dans les traités des pédagogues : le choix d'un état. Ainsi les filles bien nées que sont les nièces de la dame Picliani apprennent à s'occuper décemment avant leur mariage (des filles du peuple s'assureraient un avenir, car il y a trois états honnêtes : l'état de religieuse, celui d'épouse, celui de travailleuse ${ }^{57}$ ). Cette conception qui associe mariage et jeunes filles de bonne famille est bien ancrée dans l'esprit des hommes du xviII ${ }^{\mathrm{e}}$ siècle ${ }^{58}$. Ganganelli, décidément bien peu novateur, la reconduit : « Le mariage est la condition naturelle de tous les hommes " propos de la gente féminine. Soulignons que c'est aussi le mariage qu'il indique au jeune comte revenu des errances, en le conseillant sur le choix d'une demoiselle ${ }^{60}$.

34 Le discours de Ganganelli est cependant libéral puisqu'il préconise de ne pas forcer la volonté des jeunes gens ${ }^{61}$. Les femmes apprécieront ce motif largement relayé par les 
romans qui, à la même époque, déclinent sous différentes formes les topoi du mariage forcé ou arrangé et ceux des déboires des époux qui se sont choisis librement au dam $\mathrm{du}$ consentement parental ${ }^{62}$. Toutefois, le mariage des nièces reste encadré, par crainte qu'un éventuel manque de discernement n'entraine une mésalliance. Est-ce là une nouvelle expression d'une forme d'incapacité féminine, préjugé dont tout homme d'alors a du mal à se départir?

Le choix d'un état fait l'objet d'un développement plus nourri dans la lettre LXXIV. Ganganelli, en bon gouverneur, préconise d'employer une année au moins à élaborer une décision, qui sera raisonnable et choisie selon les inclinations du jeune homme ${ }^{63}$. Là encore la volonté ne sera pas forcée mais prédisposée par des conversations avisées et fréquentes.

36 Ces principes reconduisent in fine l'ordre social établi. La pédagogie, c'est-à-dire les méthodes et principes de transmission des savoirs (connaissances, capacités et attitudes), est peut-être plus novatrice. Trois entrées nous permettront de répondre.

Le pseudo-Ganganelli insiste à plusieurs reprises sur la nécessité de "proportionner » ses préceptes et conseils à l'intelligence et à l'âge du pupille. Si ce concept a été bien étayé par Rousseau, résumé à cette maxime fameuse : « il faut considérer l'homme dans l'homme et l'enfant dans l'enfant $»^{64}$, il n'en est pas moins attesté par ailleurs, appartenant au nombre des usages raisonnables en pédagogie : ainsi, Locke, lorsqu'il préconisait de raisonner avec les enfants, précisait que ces raisons étaient nécessairement " proportionnées à leur capacité " ${ }^{65}$. Dans son ouvrage De l'éducation des filles, Fénelon avait intitulé le cinquième chapitre «Instructions indirectes : il ne faut pas presser les enfants ${ }^{66}$. Caraccioli adopte cette position, tant pour les demoiselles ${ }^{67}$ que pour les jeunes gens pour lesquels viendra le moment où le père leur parlera " en ami des néants des plaisirs dont le monde fait sa félicité", pour mieux les en prémunir ${ }^{68}$.

38 À chaque âge ses pratiques, à chaque heure également, car le pédagogue Ganganelli invite à ménager des récréations, repos effectif ou alternance dans les travaux intellectuels ${ }^{69}$. S'il est peut-être inspiré par ses anciens maitres de l'Oratoire, il développe cependant une pédagogie dont les principes semblent assez proches de ceux de Mme d'Épinay par exemple, tels que Marianne Charrier-Vozel les identifie: «souplesse et pragmatisme » en sont deux mots d'ordre, promenades et conversations, les modalités pratiques ${ }^{70}$. L'ensemble vise à établir un climat ludique, qui n'exclut pas la fermeté parce qu'il faut être, selon le pseudo-Ganganelli, «ami de l'ordre $»^{71}$. Il en résulte surtout une intimité entre l'enseignant et l'enseigné ${ }^{72}$. Il faut cependant nuancer en précisant que cette recommandation pédagogique n'est pas nouvelle: Crousaz conseille de "finir la leçon avant même que l'attention soit épuisée et se lasse $»^{73}$; Vaubrières, dans les Principes sur l'éducation de la noblesse, conseille également de « prévenir l'ennui » et, pour cela, de « varier les occupations » ${ }^{74}$. On s'accorde alors pour dépriser les éducations trop coercitives ${ }^{75}$.

39 Le pseudo-Ganganelli insiste enfin sur les valeurs de l'exemple, l'imitation étant un élément essentiel de sa conception de la transmission des savoirs : l'enfant aura envie d'imiter son gouverneur, il aura à cœur de reproduire la sagesse maternelle ; il voudra à son tour reconduire la bonté paternelle. Locke souligne que tout devient à charge dès que les enfants voient qu'on les oblige par devoir ${ }^{76}$. Ainsi, le sentiment doublé de raison est le nerf d'une telle conception pédagogique : la sagesse emporte la confiance, la confiance gagne les cœurs, les cœurs gagnent les esprits. Vos filles «aimeront la 
religion, si par votre exemple et par votre douceur, vous savez la faire aimer $»^{77}$, dit Ganganelli à la tante; "quand un fils est persuadé qu'un père ne lui parle que raison, [...] il écoute ; et les avis ont le meilleur effet ", souligne-t-il à l'intention du père de famille ${ }^{78}$. Le trait rappelle cette persuasion par la douceur dont Fénelon s'était fait le chantre. Dès lors, les méthodes pédagogiques de Ganganelli semblent appliquer ce principe dans l'ordre, non de la conversion des hétérodoxes, mais de la pédagogie infantile. Mais est-ce pour autant remarquable? Sénèque, adressant à Lucilius des lettres de direction philosophique, écrivait déjà : «La voie des préceptes est longue, celle des exemples courte et efficace $\aleph^{79}$. Chez Locke aussi était soulignée la nécessité d'y recourir :

De tous les moyens qu'on doit employer pour instruire les enfants, il n'y en a point de plus simple, de plus aisé ni de plus efficace que de leur mettre devant les yeux des exemples des choses que vous voulez leur faire pratiquer ou éviter. ${ }^{80}$

Quant au ressort affectif, il était déjà à l'œuvre dans la pédagogie oratorienne soucieuse d'offrir une éducation à laquelle «on peut adhérer par le cœur et qui permette à l'enfant de se trouver heureux et en accord avec lui-même $»^{81}$ : en somme, conformément à la maxime de Bérulle, «toute l'autorité est dans la douceur " $^{82}$.

41 Le pédagogue qui s'exprime à travers ces lettres, petits traités d'éducation adressés, ne s'est donc pas défait du déterminisme naturel qui légitime l'inégalité des sexes, ni de cet état de fait qui réserve l'éducation à une jeunesse fortunée. En ne revendiquant nullement pour les femmes un droit au savoir et à la citoyenneté (ces considérations sont réservées à la frange masculine de l'espèce humaine), il entérine un état politique et social qui est celui de l'Ancien Régime. Les propositions et finalités qui sont prêtées à Ganganelli sont marquées d'un certain conformisme. Toutefois, sa pédagogie est assurément de celles qui se fondent sur une psychologie de l'enfant, étant en cela plus éclairée que d'autres. Elle est sans doute le fruit des leçons de la fonction de précepteur et de gouverneur qu'exerça Caraccioli et de ses nombreuses lectures, nourrie de la tradition et ouverte aux idées nouvelles, sans que l'alliance indéfectible de l'éducation et de la religion ne soit jamais récusée.

42 La figure du maitre, directeur et éducateur, est ainsi un trait essentiel du personnage que crée Caraccioli en prêtant des lettres au défunt Clément XIV. Son entreprise, si elle est assurément mercantile et fondée en cela sur une bonne analyse du marché éditorial et de ses potentialités, est aussi une entreprise d'hagiographie catholique. Il s'agit de promouvoir la personne de Clément XIV, d'en faire une figure de pape exemplaire, discours propre à couper court aux calomnies et à édifier ces fidèles qu'il faut reconquérir, à une époque où l'incrédulité fait rage.

Quoi de plus efficace que de donner à entendre la voix d'un maître par le biais des lettres qu'il aura adressées, dans la sphère privée, à des correspondants divers? Quoi de plus crédible également? En effet, la figure du maitre s'accorde avec l'horizon d'attente du public s'agissant d'un religieux devenu pontife. Sont nombreuses en effet les vies de saints et de bons pères qui consacrèrent leur existence à l'étude. La figure d'un saint Jérôme, père de l'Église, dirigeant par lettres des éducations chrétiennes (nous songeons aux épîtres à Laeta et Gaudentius), était propre à créditer la construction ganganellienne d'un halo d'authenticité. Le souvenir de saint François de Sales, écrivant à Philotée les lettres qui forment l'Introduction à la vie dévote, pouvait concourir au même effet. La correspondance de Fénelon, celle de Duguet constituaient des modèles antérieurs. Cette image, véritable préjugé, se devait donc d'être actualisée 
dans le recueil de lettres si Caraccioli voulait qu'il trouve crédit. Inscrivant la figure de Ganganelli dans une lignée implicite de religieux dirigeant à distance l'éducation chrétienne de leurs correspondants, Caraccioli lui octroyait un semblant supplémentaire de vérité.

Le topos du docte maître constitue un autre élément essentiel des supercheries puisque cette figure contribue à former celle de l'écrivain supposé, instance nécessaire à la crédibilité du recueil. De fait, pour que ces lettres émanent de Clément XIV, il est nécessaire que ce dernier possède au préalable (comme condition de possibilité) les qualités du maître, qui maîtrise le langage, les codes et les savoirs. De plus, la récurrence du motif du pédagogue participe à l'unité du portrait, à l'élaboration d'un caractère, éléments nécessaires à la consistance de la figure de papier que Caraccioli propose comme modèle à suivre.

En outre, la forme épistolaire, dans l'ordre profane ou religieux, avait déjà trouvé ses modes d'application, la littérature d'éducation ayant repéré dans cette forme spécifique (un discours en "je» adressé à un "vous») "un moyen efficace de dispenser un programme pédagogique en évitant le didactisme des traités jugés le plus souvent fastidieux. $\|^{83}$ De surcroît, ce discours épistolaire, plus malléable, plus souple, peut se placer à la croisée des registres, autorisant la juxtaposition de contenus variés qui rendront la leçon moins aride et entraîneront l'intérêt. Le dialogisme qui préside à la rédaction de la lettre, et que Caraccioli a soin d'introduire pour garantir l'effet de réel, n'est pas non plus à minorer quand il s'agit de démontrer que la forme lettre est bien propre à véhiculer des idées. C'est en somme une formule autant qu'un moyen de transmettre des opinions au plus près du cœur, le lecteur véritable étant d'autant plus atteint que les destinataires premiers forcent son identification. Bref, le discours épistolaire s'insinue dans l'âme des lecteurs. Sa brièveté, autre caractéristique générique, justifie la simplification. Enfin, la lettre autorise la production d'un énoncé intime, amical et autoritaire, à même de faire impression sur les lecteurs.

Deux traces de réception des Lettres intéressantes confirment ces analyses. La première émane de Louis-Sébastien Mercier qui mentionne les Lettres de Clément XIV dans le Tableau de Paris :

Il faut beaucoup de livres, puisqu'il y a beaucoup de lecteurs. Il en faut pour toutes les conditions, qui ont un droit égal à sortir de l'ignorance. [...] S'il n'y avait que les ouvrages des La Bruyère, des Montesquieu, des Boulanger, des Buffon, des Rousseau, la multitude ne pourrait être éclairée. Ces livres sont trop substantiels, il lui faut une nourriture plus légère et plus détaillée. Ôtez les livres médiocres, et l'on ne saura bientôt plus lire ni distinguer les bons. Les lettres fictives du pape Ganganelli ont eu un succès prodigieux. Toutes les idées qu'elles renferment sont communes ; mais ces idées sont bonnes, claires, facilement exprimées. La multitude a été enchantée de l'ouvrage, et a dû l'être. C'est toujours un échelon de monté ; et d'après ce succès, que les sots journalistes n'ont pas assez remarqué, il sera plus facile de la conduire à quelque ouvrage élevé. ${ }^{84}$

Mercier salue la formule épistolaire, mêlant proximité et rigueur, familiarité et civilité, réceptacle idéal pour les maximes de sagesse du pseudo-Ganganelli, pour ses plans de conduite. La simplification, alliée à une écriture qui place à son fondement le rapport de personne à personne, est désignée pour un mode adéquat de transmission des savoirs.

Les lecteurs qu'évoquent les rédacteurs de la Bibliothèque $d u$ Nord, un périodique émanant de l'espace rhénan, corroborent les analyses de Mercier. Précisons que la 
recension est écrite après que la mystification a été éventée et que le véritable auteur, Caraccioli, a été reconnu pour tel.

Un grand nombre de personnes pieuses [...] auraient souhaité qu'on les laissât dans une erreur, que leur respect pour le chef de l'Église leur rendait chère et même utile. Ses lettres les édifiaient, les consolaient, leur traçaient des maximes sages, des plans de conduite aisés à suivre; elles leur développaient la morale évangélique d'une manière propre à la faire aimer..$^{85}$ sont ceux qu'a imprimés l'auteur du Véritable Mentor.

Le père Ganganelli, dans une lettre adressée à un jeune seigneur, lui trace un plan de bibliothèque. Il lui recommande la lecture de Bossuet sur l'histoire universelle, les Pensées de Pascal sur les vérités de la religion, les Annales d'Italie par Muratori, Virgile, Horace, etc., etc., etc. M. Caraccioli, dans son Véritable Mentor, donne une idée d'une semblable bibliothèque, et nomme précisément les mêmes auteurs et les mêmes ouvrages qu'indique le père Ganganelli. Il me serait facile, Monsieur, de vous prouver cette parfaite égalité en rapprochant les morceaux des deux ouvrages. Mais ces sortes de citations, après toutes celles que j'ai déjà mises sous vos yeux, deviennent inutiles, et finiraient peut-être par vous ennuyer. ${ }^{89}$

Voici pourtant les pensées que le même journaliste suppose ensuite à Caraccioli à propos de son personnage :

Rien de si aisé que de le faire écrire. C'était un religieux, un cardinal d'un esprit cultivé, adonné aux sciences, surtout à la théologie dont il a été professeur ; [...] Je puis, avec ces qualités, le mettre en relation avec bien des personnages, et le faire parler de tout à peu près. [...] il écrira sur la vie religieuse, sur la solitude, sur la théologie; il traitera dans plusieurs lettres des grandes vérités de la religion; il donnera des plans pour combattre les incrédules modernes, etc. Savant, [...] il fera des lettres sur la physique, et sur les mathématiques, sur la politique, sur l'esprit des divers gouvernements, sur la géographie, sur l'histoire moderne ; [...] il écrira sur la poésie, sur l'éloquence, sur l'histoire, sur le choix des livres pour former une bibliothèque, sur les arts, sur la peinture, la sculpture, l'architecture, etc. Plein de douceur et d'aménité, il fera une conversion célèbre dans la personne d'un jeune comte, etc. Il me sera d'autant plus facile de composer ces lettres, que j'ai traité moimême tous ces objets. [...] Religion, morale, dogme, théologie, philosophie, sciences, beaux-arts, poésie, éloquence, histoire, politique, mathématiques, littérature, 
éducation, voyage, etc. Voilà les matières sur lesquelles on trouve tant de chapitres dans mes divers ouvrages; je redonnerai les mêmes choses sous le nom de Ganganelli. ${ }^{90}$

Le critère de l'intertextualité comme marqueur de l'inauthenticité est encore activé par l'auteur du Tartuffe épistolaire démasqué: à propos de la lettre VI adressée à une religieuse carmélite, il note, non sans raillerie: «Le Ganganelli qui a fait cette lettre avait eu la précaution, auparavant, de faire sa lecture spirituelle dans quelques sermonnaires français. $»^{91}$ Le jésuite insinuait ainsi que Caraccioli avait puisé sa matière dans les secrétaires et autres « livres pratiques».

5 La lettre-précepte, si commode pour véhiculer les principes et valeurs d'un christianisme bienfaisant incarné par sa figure tutélaire, le pape Ganganelli revu et corrigé par Caraccioli, trouve ses limites au moment même où elle actualise sa puissance. Cette puissance, que $M$. de Certeau repérait dans les panégyriques de saints $^{92}$, permet de formuler cet effet propre à la lettre lorsqu'elle se double d'un épistolier volontiers conseilleur, sans connotation péjorative. Nous soulignerons donc, non seulement la puissance de cet écrit adressé (dans lequel les conseils destinés au destinataire premier, surnuméraire ou historique, s'insinuent dans l'âme du lecteur, auquel il s'adresse par effet de consécution), la puissance de ce recueil qui véhicule une image exemplaire du sacerdoce, celle de la lettre donc, qui par le rapport de personne à personne qu'elle implique et construit, apparaît comme l'une des formules les plus propres à l'exercice d'un "gouvernement " bien entendu. Mais Caraccioli, trop enclin à céder à la facilité ou à court d'inspiration, n'a pas su donner aux lettres prêtées au pape l'originalité qui l'aurait gardé du discrédit.

Le topos du maître est un rouage fondamental de la fraude pieuse qu'a forgée Caraccioli, mais aussi, sous un autre point de vue et selon d'autres modalités de lecture, un marqueur d'inauthenticité. L'étude des formes que ce topos revêt dans le recueil a d'abord permis de mettre en lumière, sur le plan générique ou formel, une logique de transposition, propre au champ épistolaire : la lettre pédagogique fonctionne comme la réplique laïque de la lettre de direction spirituelle.

S'agissant des contenus éducatifs, notre étude confirme les analyses de Martine Jacques à propos de la doxa caracciolienne. Elle apporte toutefois des compléments : le sage Ganganelli des Lettres intéressantes professe en matière d'éducation des conseils éclairés, fondés sur une psychologie de l'enfant et une pédagogie adaptée, mais toujours et pleinement orientés vers une philosophie chrétienne inscrite dans une société d'ordres. Il ne s'agit pas de faire de Ganganelli un philosophe et encore moins un pédagogue à la manière de Rousseau, mais plutôt de ramener, par le truchement de ce grand épistolier, les usages raisonnables dans le giron de la religion, au service de l'ordre social établi.

58 En termes d'axiologie enfin, la surreprésentation de la figure du maître et sa participation à l'opération hagiographique attestent sa valorisation. Ces lettres sapientiales sont le lieu idéal pour l'expression des nombreuses qualités de Ganganelli. Bon, sage, humain, profondément orthodoxe, il incarne les attributs du bon gouverneur tels que Caraccioli les brossait déjà dans le Véritable Mentor. Enfin, ces qualités, réunies, contribuent à l'aura du Saint-père, bon père pour tous. 
59 La formule retenue par Caraccioli pour assurer une transmission maximale des préceptes, une fraude pieuse, semblait donc la bonne. Il n'en demeure pas moins que les vues sur l'éducation que Caraccioli prête à Ganganelli sont les siennes, c'est-à-dire un compendium des différents modèles éprouvés durant l'âge classique, probablement la synthèse de ses expériences concrètes, mais aussi des vues qu'il suppose à même de répondre aux attentes du lectorat et d'auréoler au mieux le défunt pontife. Le topos du conseiller, sage gouverneur, père attentif, père tout à tous, décliné dans l'ordre sacré et profane, se constituait ainsi en élément-clef de la tentative hagiographique de Caraccioli : une hagiographie profane, qui sacre et le directeur spirituel et le pédagogue du siècle.

\section{ANNEXES}

[Louis-Antoine de Caraccioli], Lettres intéressantes du pape Clément XIV Ganganelli, Paris, Lottin, 1776, tome I, p. 275-278 (Lettre LV).

À la dame Picliani

Ce n'est pas une chose indifférente de garder vos deux filles avec vous : la qualité de mère vous impose les devoirs les plus importants. Le monde viendra se mettre continuellement entre vous et vos enfants, si vous n'avez pas soin de l'écarter, non avec cette austérité qui n'excite que des murmures, mais avec cette sagesse qui gagne la confiance.

Vos filles ne seront que des hypocrites, si vous les accablez d'instructions, et si vous les inquiétez ; tandis qu'elles aimeront la religion, si par votre exemple et par votre douceur, vous savez la faire aimer.

On ne conduit pas des personnes de vingt ans comme si elles n'en avaient que dix. Il est des traitements et des leçons pour les âges comme pour les conditions.

Entretenez le plus que vous pourrez le goût des bonnes lectures et du travail ; mais avec cette aisance qui n'assujettit point à la minut[i]e, et avec cet esprit de discernement, qui sait différencier d'un cloître une maison séculière.

Établissez vos filles selon leur bien et selon leur état, en ne forçant point leur volonté, à moins qu'elles ne voulussent s'allier avec des gens dissipateurs ou vicieux. Le mariage est la condition naturelle de tous les hommes : ce sont des exceptions à la règle lorsqu'on s'en dispense.

Sans aimer la mondanité, ne vous rendez point ridicule sur les usages du monde. La piété devient sujet de raillerie, quand on la présente sous des dehors singuliers : la femme sage évite de se faire remarquer.

Quand on est né pour prendre certains habits, il faut les porter, mais toujours avec la décence qui convient à la pudeur. 
Vous aurez soin que vos demoiselles fréquentent la société. La vraie dévotion n'est ni brusque ni farouche : la solitude mal entendue irrite les passions ; et pour de jeunes personnes, il est souvent plus sûr de fréquenter un monde choisi, que de rester seul. Vous inspirerez la gaieté, pour qu'on n'ait pas l'air d'y traîner la piété : vos récréations consisteront dans des promenades et dans des petits jeux ; et lorsqu'il sera question de s'appliquer, vous ne parlerez ni de ces études profondes, ni de ces sciences abstraites, qui souvent rendent le sexe vain et jaseur.

Faites-vous surtout aimer : c'est le plus grand plaisir auquel une mère puisse aspirer, et la plus grande prérogative dont elle puisse jouir, pour opérer le bien selon sa volonté.

Que vos domestiques aient de la religion et de l'honnêteté : ils sont capables de tous les crimes, quand ils ne craignent pas Dieu. On ne doit user avec eux, ni de hauteur, ni de familiarité, afin de les traiter comme des hommes et comme des inférieurs. La justice est la mère de l'ordre : tout est à sa place, quand on se comporte avec équité.

Ne punissez jamais qu'avec peine, et pardonnez toujours avec plaisir.

Fréquentez votre paroisse, pour que les brebis se trouvent souvent avec leur pasteur ; c'est conforme aux saints canons, ainsi qu'à l'usage ancien.

Votre sagesse vous apprendra le reste. Je compte beaucoup sur vos lumières et sur votre bonne volonté, comme vous pouvez être vraiment assurée de la respectueuse considération avec laquelle j'ai l'honneur d'être, etc.

À Rome, ce 15 novembre 1754

\section{NOTES}

1. «Je marie $\mathrm{M}^{\text {me }}$ Le Prince de Beaumont à $\mathrm{M}$. le marquis Caraccioli, auteur de la Conversation avec soi-même, et sans difficulté un des plus plats barbouilleurs de notre temps. Il a publié depuis peu deux volumes de Lettres récréatives et morales sur les mœurs du temps. Il nous menace d'en donner encore deux autres. $\mathrm{M}^{\mathrm{me}}$ Le Prince et $\mathrm{M}$. Caraccioli se feront par contrat de mariage un don mutuel de leurs œuvres à la décharge entière du public.» (Friedrich Melchior Grimm, Correspondance littéraire philosophique et critique par Grimm, Diderot, Raynal, Meister, etc. revue sur les textes originaux comprenant outre ce qui a été publié à diverses époques les fragments supprimés en 1813 par la censure, les parties inédites conservées à la bibliothèque ducale de Gotha et à l'Arsenal à Paris, éd. de Maurice Tourneux, Paris, Garnier frères, 1877-1882, 15 octobre 1767, tome VII, p. 461).

2. [Louis-Antoine de Caraccioli], Lettres intéressantes du pape Clément XIV, (Ganganelli), Paris, Lottin le Jeune ; Lyon, Bruyset-Ponthus ; Rouen, Bénitier, 1776, 2 vol. in-12.

3. Martine Jacques, «Vertus éducatives de l'apologétique selon L.-A. Caraccioli: éclairer l'homme, entre Grâce et Lumières ", Apologétique 1650-1802. La nature et la grâce, Nicolas Brucker (dir.), Berne, Peter Lang, 2010, p. 245-246, Maximilian Gröne: «L'utilité d'un prince. LouisAntoine Caraccioli entre Fénelon et Rousseau ", Démocratisation et diversification. Les littératures d'éducation au siècle des Lumières, Rotraud von Kulessa (dir.), Paris, Classiques Garnier, « Rencontres », 2015, p. 243-260.

4. Voir notre article «Caraccioli, Louis-Antoine de (1719-1803)», Dictionnaire des anti-Lumières et des antiphilosophes (France, 1715-1815), D. Masseau (dir.), Paris, Honoré Champion, 2017, p. 301-307.

5. Nous songeons, parmi d'autres, aux travaux de Sylviane Coppola (Albertan-Coppola), «Un apologiste mondain: le marquis Louis-Antoine de Caraccioli ", Ragioni dell' anti-illuminismo, Lionello Sozzi (dir.), Turin, Edizioni dell'orso, 1992, p.3-27, et de Philippe Lefebvre, 
"Apologétique et séduction au XVIII ${ }^{\mathrm{e}}$ siècle ", Littérature et séduction. Mélanges en l'honneur de Laurent Versini, Roger Marchal et François Moureau (dir.), Paris, Klincksieck, 1997, p. 209-216. Voir aussi notre notice, "Apologétique mondaine", Dictionnaire des anti-Lumières et des antiphilosophes (France, 1715-1815), op. cit., p. 112-121.

6. Stéphanie Géhanne Gavoty, L'Affaire clémentine. Une fraude pieuse à l'ère des Lumières, Paris, Classiques Garnier, «L'Europe des Lumières », 2014.

7. La Vie du pape Clément XIV et le supplément aux deux premiers volumes des Lettres intéressantes du pape Clément XIV (soit les volumes III et IV), qui offrent d'autres données exploitables sur ce sujet, ont été écartés du corpus.

8. Allusion à Vincent Jouve, L'Effet-personnage dans le roman, Paris, PUF, 1992.

9. Jean-François Jeandillou, Supercheries littéraires. La vie et l'œuvre des auteurs supposés [1989], éd. revue et corrigée, Genève, Droz, 2001.

10. Saint Jérôme, Lettres à Laeta et à Gaudentius : Epistola ad Laetam. De institutione filiae; Epistola ad Gaudentium. De Pacatulae infantulae educatione (Saint Jérôme, Sancti Eusebii Hieronymi Stridonensis presbyteri opera omnia [...] accurante et denuo recognoscente J.-P. Migne [...], Patrologiae cursus completus omnium SS. Patrum, doctorum scriptorumque ecclesiasticorum sive Latinorum, sive Graecorum, Patrologia Latina, J.-P. Migne, Brepols, Turnholt [Belgique], 1986 [fac-sim. de l'éd. de Paris : J.-P. Migne, 1845-1846], tome. XXII, p. 677-689, 1095-1099.

11. Nous songeons aux échanges du saint avec Jeanne de Chantal, dont la correspondance a fait l'objet d'une édition critique récente: François de Sales, Jeanne de Chantal, Correspondance François de Sales et Jeanne de Chantal, David Laurent (dir.), Paris et Perpignan, Desclée de Brouwer, 2016.

12. Johann Heinrich Samuel Formey, Principes élémentaires des belles-lettres, nouvelle édition, Amsterdam, J. H. Schneider, 1763, p. 185.

13. À propos des lecteurs des Lettres intéressantes du pape Clément XIV, voir S. Géhanne Gavoty, L'Affaire clémentine, op.cit., p. 216-227. La citation est tirée de [Jacques-Julien Bonnaud], Le Tartuffe épistolaire démasqué, ou épître très familière à M. le marquis Caraccioli, colonel (in partibus), éditeur, et comme qui dirait auteur des lettres attribuées au pape Clément XIV (Ganganelli), etc., Liège, s. n., 1777, p. 509.

14. [L.-A. de Caraccioli], Lettres intéressantes, op.cit., tome I, p. XIV. L'objet était effectivement d'importance comme en témoigne le privilège du Véritable Mentor, octroyé en 1759 : «Rien n'est plus important à un père de famille, que de donner bonne éducation à ses enfants; car un fils bien élevé le consolera et deviendra les délices de son âme : Erudi filium tuum, et refrigerabit te, et dabit delicias animae tuae. Prov. 29. Au lieu qu'un fils mal instruit sera sa honte : Confusio patris est de filio indisciplinato. Eccl. 22. [...] Les nobles seigneurs donc, et autres qui ont les facultés convenables, ont intérêt de choisir à leurs enfants des gouverneurs capables, qui par leur conduite, prudence, sagesse, piété et entretiens, puissent perfectionner ce que les précepteurs ont ébauché dans leurs études. »

15. [J.-J. Bonnaud], Le Tartuffe épistolaire démasqué, op. cit., p. 1.

16. Voir Pauline Chaduc, Fénelon, direction spirituelle et littérature, Paris, Champion, «Lumières classiques ", 2015 et Viviane Mellinghoff-Bourgerie, «L'écrivain au service des âmes : tradition et avatars de l'épistolarité spirituelle », Travaux de littérature, «La spiritualité des écrivains », XXI, 2008, p. 117-130.

17. P. Chaduc, Fénelon, direction spirituelle et littérature, op.cit., p.141. M. Gröne précise toutefois que le gouverneur a la charge de la formation morale de son disciple (M. Gröne, "L'utilité d'un prince ", article cité, p. 145).

18. [L.-A. Caraccioli], Lettres intéressantes, op.cit., lettre XCVII, tome II, p. 95.

19. Ibid., p. 39-53.

20. Ibid., p. 117-131. 
21. V. Mellinghoff-Bourgerie, "L'écrivain au service des âmes", article cité, p. 127-128 ; Marianne Charrier-Vozel, « Des remerciements aux lettres de conseils dans la correspondance de $\mathrm{M}^{\mathrm{me}}$ Riccoboni », dans Conseiller, diriger par lettre, Élisabeth Gavoille et François Guillaumont (dir.), 2017, Tours, Presses universitaires François-Rabelais, « Perspectives littéraires », p. 553-554.

22. Mentionnons cependant l'Émile de Rousseau, les lettres d'éducation de $\mathrm{M}^{\text {me }}$ d'Épinay (les Lettres à mon fils sont en effet douze lettres, datées du $1^{\text {er }}$ octobre 1758 , que $\mathrm{M}^{\text {me }}$ d'Épinay écrit à son fils pour accompagner son éducation de ses préceptes en la matière) ou Adèle et Théodore. Lettres sur l'éducation contenant tous les principes relatifs aux trois différents plans d'éducation des princes et des jeunes personnes de l'un et l'autre sexe par $\mathrm{M}^{\mathrm{me}}$ de Genlis (1782).

23. Un parallèle avec Le Comte de Valmont ou les égarements de la raison de l'abbé Philippe-Louis Gérard (1774) peut être esquissé : dans ce roman apologétique, les lettres se défont de la brièveté associée au genre pour virer à la suite de conférences (Voir Nicolas Brucker, Littérature et apologétique: le cas de l'abbé Philippe-Louis Gérard (1731-1813), thèse de doctorat sous la dir. de Sylvain Menant, Paris, Université de Paris-IV, 2000, notamment p. 69).

24. L.-A. de Caraccioli, Le Véritable Mentor, ou l'éducation de la noblesse, par le marquis Caraccioli, colonel au service du roi de Pologne, électeur de Saxe, Liège, Bassompierre ; Francfort, en foire ; Bruxelles, J. Vanden Berghen, 1759, p. XII.

25. Marie-Frédérique Pellegrin, «La Ferté-Imbault contre D’Alembert. Résistance mondaine et intellectuelle aux Lumières ", CEuvres et critiques, XXXVIII, L'Apologétique littéraire et les antiLumières féminines, p. 139.

26. [L.-A. de Caraccioli], Lettres intéressantes, op. cit., tome I, p. 144-162.

27. Ibid., p. 103-104 (lettre XX).

28. Ibid., p. 145.

29. Marie-Emmanuelle Plagnol-Diéval, "Que lire et comment lire. Quelques prescriptions au $\mathrm{XVIII}^{\mathrm{e}}$ siècle ", Démocratisation et diversification, op. cit., p. 65-80.

30. [J.-J. Bonnaud], Le Tartuffe épistolaire démasqué, op.cit., p. 211. Le rédacteur de L'Année littéraire, dans son article daté du 20 décembre 1775, avait qualifié les lettres au jeune comte de "petit roman de spiritualité » (Élie-Catherine Fréron, Année littéraire, [Paris, Le Jay], 1775, tome VIII, p. 318).

31. M. Gröne, «L'utilité d'un prince », article cité, p. 145.

32. Voir également $\mathrm{S}$. Géhanne Gavoty, «Le mythe des Lumières à propos d'un "anti-Lumières" : Clément XIV revu et corrigé ", Les Philosophes et leurs papes, Actes du colloque Les papes imaginaires des Lumières françaises. 1713-1789, Academia Belgica Rome, 13-15 mars 2008, Jan Herman, Kris Peeters et Paul Pelckmans (dir.), Amsterdam, Rodopi, « Faux titre », n 337, 2009, p. 215-229.

33. Martine Jacques, «Vertus éducatives de l'apologétique selon L.-A. Caraccioli », article cité, p. 249.

34. Marie-Emmanuelle Plagnol-Diéval, "Que lire et comment lire. Quelques prescriptions au XVIII ${ }^{\text {e }}$ siècle ", article cité, p. 68: "les auteurs de plans de lecture [...] ne justifient pas la distinction entre filles et garçons, tant l'idée est communément admise ».

35. Roger Chartier, Dominique Julia, Marie-Madeleine Compère, L'éducation en France $d u X^{e} I^{e}$ au XVIII siècle, Paris, Sedes, 1976, p. 231-247 (Chap. VIII : L'éducation des filles).

36. Nous songeons, entre autres, à La Religieuse de Diderot.

37. [L.-A. de Caraccioli], Lettres intéressantes, op.cit., tome I, p. 375.

38. Ibid., p. 376.

39. Roger Chartier, Dominique Julia, Marie-Madeleine Compère, op.cit., p. 232. Marianne Charrier-Vozel acte également le rôle de gardienne du foyer toujours assigné à la femme (M. Charrier-Vozel, « Lettre et éducation. Des manuels épistolaires à Louise d'Épinay, Marie-Élisabeth de La Fite et Stéphanie Félicité de Genlis ", Démocratisation et diversification, op. cit., p. 91). MarieOdile Bernez signale la permanence de ce modèle lorsqu'elle étudie le cas d'Hannah More («Éducatrices anglaises dans la tourmente révolutionnaire : infléchissement des pratiques et des 
discours à la fin du XVIII siècle ", Femmes éducatrices au siècle des Lumières, Isabelle Brouard-Arens et Marie-Emmanuelle Plagnol-Diéval (dir.), Rennes, Presses universitaires de Rennes, 2007, p. 328).

40. [L.-A. de Caraccioli], Lettres intéressantes, op. cit., tome I, p. 277 (et ci-dessous).

41. Martine Sonnet, L'éducation des filles au temps des Lumières, Paris, Les éd. du Cerf; CNRS éd., 2011, p. 233.

42. Gabriel Campyaré, Histoire critique des doctrines de l'éducation en France depuis le seizième siècle, cinquième édition, Paris, Hachette, 1885, p. 333.

43. [L.-A. de Caraccioli], Lettres intéressantes, op. cit., t. I, p. 14 : « Vous [...] trouverez [à Bologne] les sciences familières au sexe même, se produisant avec dignité dans des écoles et dans des académies, où chaque jour on leur érige des trophées. »

44. Ibid., p. 203-209 (lettre XXXVIII).

45. Ibid., p. 377 (lettre LXXIV).

46. Jean-Jacques Rousseau, Émile ou de l'éducation, Cuuvres complètes, éd. de Charles Wirz et Pierre Burgelin, Paris, Gallimard, «Bibliothèque de la Pléiade», 1969, tome IV, livre III, p. 470-477 («Apprends un métier», p. 470) et p. 478-480 (choix du métier), Locke, De l'éducation des enfants, nouvelle édition, Londres/Paris, Servière, 1783, tome II, p. 263-264, 268, 279.

47. Dominique Julia, «Entre sacré et savoir : l'Oratoire au XVIII ${ }^{\mathrm{e}}$ siècle », Le Collège de Riom et l' enseignement oratorien en France au XVIII e siècle, Paris/Oxford, CNRS Éditions/Voltaire Foundation, 1993, parmi d'autres p. 308-309 (organisation raisonnée des études), p. 321-322 (la pédagogie par le jeu); Gilbert Py, Rousseau et les éducateurs. Essai sur la fortune des idées pédagogiques de J.-J. Rousseau en France et en Europe au XVIII siècle, Oxford, Voltaire Foundation, « Studies on Voltaire and the eighteenth century », 356, 1997, p. 124.

48. G. Campyaré, op. cit., p. 355 : «[Fénelon] admet la poésie et l'éloquence, à condition qu'on écarte tout ce qui est dangereux pour la pureté des mœurs »; à propos de l'influence de Fénelon sur Caraccioli, voir notre article à paraître: «Fénelon à l'aune de l'apologétique mondaine (1760-1770) », Lectures et figures de Fénelon (actes du colloque de Cambrai et Paris, 9-12 septembre 2015), Paris, PUPS.

49. Pierre Mesnard, « Rollin forge l'esprit de l'enseignement secondaire », Les Grands pédagogues, Jean Château (dir.), Paris, PUF, 1980 (6 édition), p. 174-175.

50. Roger Chartier, Dominique Julia, Marie-Madeleine Compère, op.cit., p. 244.

51. [L.-A. de Caraccioli], Lettres intéressantes, op.cit., tome I, p. 370.

52. Ibid.

53. «Ce n'est point par des pratiques minutieuses que vous ferez de vos enfants de vrais chrétiens. Le christianisme est le plus grand ennemi du pharisaïsme et de la superstition. » (Ibid., p. 371)

54. Une lettre, la dixième, aborde cette question : Ganganelli met en garde une correspondante anonyme contre une dévotion ostentatoire et scrupuleuse à laquelle il oppose une piété véritable et profonde (Ibid., p. 57-65).

55. «La critique de la fausse dévotion, lieu commun du discours moral à l'âge classique, sert par un mouvement de balancier à d'autant plus louer la piété domestique, façon de défendre la foi chrétienne sur la question de sa conciliation avec les voies du siècle, en chassant de l'esprit du lecteur toute représentation négative pour imposer par contraste une image douce et aimable. Caraccioli condamne ainsi la dévotion "pharisaïque", qui n'est que "momeries", "caprices", "extravagances": "Nous voyons des hommes invoquer perpétuellement les saints, se prosterner devant toutes leurs images, et à peine prier Jésus-Christ, et ne le pas distinguer de ses créatures qui n'ont de mérite que ses dons." " (Nicolas Brucker, "Julie dans la gloire des saints. Construction/déconstruction du modèle de la piété ordinaire », 2017, p. 2. <hal-01482994>) 
56. Alicia C. Montoya, «Madame Leprince de Beaumont et les "Lumières religieuses" ", Madame Leprince de Beaumont; De l'éducation des filles à La Belle et la Bête, Jeanne Chiron et Catriona Seth (dir.), Paris, Classiques Garnier, 2013, p. 135.

57. M. Sonnet, op. cit., p. 234 et 263.

58. Ibid., p. 271.

59. [L.-A. de Caraccioli], Lettres intéressantes, op. cit., tome I, p. 275 et ci-dessous (lettre LV).

60. Ibid., p. 154 (lettre XXX) et p. 345 (lettre LXVIII).

61. Ibid., p. 275 (et ci-dessous) : «Établissez vos filles selon leur bien et selon leur état, en ne forçant point leur volonté [...]» (lettre LV).

62. Collette Piau-Gillot, "Violences et mariage à la fin du siècle des Lumières ", Violence et fiction jusqu'à la Révolution: travaux du IXe Colloque international de la Société d'analyse de la topique romanesque, Milwaukee-Madison, septembre 1995, Martine Debaisieux et Gabrielle Verdier (dir.), Tübingen, G. Narr, 1998, p. 393-402.

63. [L.-A. de Caraccioli], Lettres intéressantes, op. cit., tome I, p. 386-389.

64. Rousseau, op. cit., livre II, p. 303.

65. Locke, op.cit., section LXXXIV «Il faut convaincre les enfants par voie de raisonnement » (tome I, p. 221-224).

66. Fénelon, CEuvres, éd. de Jacques Le Brun, Paris, Gallimard, « Bibliothèque de la Pléiade », 1983, tome I, p. 102. Une réflexion proche est prêtée à Ganganelli : «On dégoûte souvent les jeunes personnes de la piété, par la raison qu'on leur demande une trop grande perfection [...] 》 ([L.-A. de Caraccioli], Lettres intéressantes, op. cit., tome I, p. 61 ; lettre X).

67. «On ne conduit pas des personnes de vingt ans comme si elles n'en avaient que dix. Il est des traitements et des leçons pour les âges comme pour les conditions. » (Ibid., p. 275, et ci-dessous)

68. Ibid., p. 385.

69. "Vous aurez soin de ménager des repos dans le travail, pour ne pas lasser la mémoire et l'esprit de vos enfants [...] Ne manquez jamais de faire succéder un livre amusant à un livre sérieux, et d'entremêler la poésie avec la prose. » (Ibid., p. 377-380)

70. M. Charrier-Vozel, « Lettre et éducation », article cité, p. 91.

71. [L.-A. de Caraccioli], Lettres intéressantes, op. cit., tome I, p. 391.

72. M. Charrier-Vozel souligne les vertus de cette intimité bienveillante («Lettre et éducation », article cité, p. 83).

73. Jean-Pierre de Crousaz, Traité de l'éducation des enfants, La Haye, V. Vaillant et Prévost, 1722, tome I, p. 205.

74. De Vaubrières, Principes sur l'éducation de la noblesse concernant les bonnes mœurs, la religion et les sciences [...] par de Vaubrières, [...] Nouvelle édition, augmentée d'un troisième volume, qui donne des notions détaillées sur les sciences et sur la poésie en particulier, Liège, chez l'auteur, 1761, tome I, p. 60. 75. Voir Martine Jacques, «Vertus éducatives de l'apologétique selon L.-A. Caraccioli », article cité, p. 255.

76. Locke, op. cit., tome I, p. 196.

77. [L.-A. de Caraccioli], Lettres intéressantes, op. cit., tome I, p. 274-275 (et ci-dessous).

78. Ibid, p. 386.

79. Élisabeth Gavoille, « La force de l'exemple dans les Lettres à Lucilius », dans Élisabeth Gavoille et François Guillaumont (dir.), op. cit., p. 284-285.

80. Locke, op. cit., tome I, p. 221.

81. Martine Jacques, «Vertus éducatives de l'apologétique selon L.-A. Caraccioli », article cité, p. 247.

82. Ibid., p. 253.

83. M. Charrier-Vozel, « Lettre et éducation », article cité, p. 83.

84. Louis-Sébastien Mercier, Tableau de Paris, éd. de Jean-Claude Bonnet, Paris, Mercure de France, 1994, tome I, p. 351. 
85. Bibliothèque du Nord, ouvrage destiné à faire connaître en France tout ce que l'Allemagne et le Nord produisent d'intéressant [...], par la Société patriotique de Hesse-Hombourg. Dédié à [...] Monseigneur le Landgrave de Hesse-Hombourg, Paris, Quillau, mars 1778, p. 200-201.

86. [J.-J. Bonnaud], Le Tartuffe épistolaire démasqué, op.cit., p. 93.

87. François Caradec, La Farce et le sacré (1977) cité par J.-F. Jeandillou, Supercheries littéraires, op. cit., p. 493.

88. Ibid., p. 482.

89. Élie-Catherine Fréron, op. cit., p. 310.

90. Ibid., p. 315-317.

91. [J.-J. Bonnaud], Le Tartuffe épistolaire démasqué, op. cit., p. 45.

92. «Puissance du récit. Il se construit sur un espace qui prend valeur utopique et exemplaire, l'unité biographique. [Charles Borromée, dans les Vies] y dresse la figure d'un prince en qui se réalise le rêve d'un siècle de chrétienté [...]». Cette "actio rhétorique " est un "exemplum séducteur ». (Michel de Certeau, Le lieu de l'autre : histoire religieuse et mystique, éd. de Luce Giard, Paris, Gallimard, Le Seuil, « Hautes études », 2005, p. 116)

\section{RÉSUMÉS}

L'auteur étudie la figure du maître dans la supercherie épistolaire conçue par Louis-Antoine de Caraccioli autour du défunt Clément XIV. Y sont étudiées les lettres pédagogiques de ce recueil dans la configuration d'ensemble où elles prennent place (direction et éducation), mais également sous l'angle des thématiques spécifiques qui y sont déployées : éducation des filles, des garçons ; lieu des études ; nature et modalités de celles-ci ; choix d'un état, etc. Il s'agit de mettre en lumière une éducation marquée par un certain conformisme, ne récusant jamais l'alliance indéfectible de la religion et de l'éducation, mais ouverte à la modernité, au moins sur le plan pédagogique. L'analyse a aussi pour but de montrer que cette figure du maitre est un rouage essentiel de la réussite de la supercherie de Caraccioli, mais aussi de son éventement.

The article speaks about the "topos" of Master in the apocryphal collection about the Pope Clement XIV published by Caraccioli. The letters on upbringing are considered to evaluate their places in the collection (direction and education). Their main topics are specified to: what are the views of those letters about girl's and boy's upbringing (gender education?)? what place of study for pupils? How to do to select a condition and a trade? etc. The conformism of this positions has been highlighted: religion and education are always interconnected; the social order of France's old regime is never contested, but the pedagogy is modern. At last, the authoress demonstrates that the topos of the Master is essential for the success of the mystification (credibility) but also his failure.

\section{INDEX}

Keywords : Caraccioli, education, epistolarity, eighteenth century, mystification, gender studies

Mots-clés : Caraccioli, éducation, épistolaire, xviiie siècle, mystification, genre 
AUTEUR

STÉPHANIE GÉHANNE GAVOTY

Sorbonne Université, CELLF 16-21 - UMR 8599 